\title{
A link between heart \& dental health
}

\author{
Yogeshwari Krishnan ${ }^{1, *}$, Anubha Agarwal ${ }^{2}$, Jyotsna Seth ${ }^{3}$ \\ ${ }^{1}$ Lecturer, Seema Dental College \& Hospital, Rishikesh, Uttarakhand, ${ }^{2}$ Assistant Professor, Hospital Administration, Deputy \\ Medical Superintendent, All India Institute of Medical Sciences, Rishikesh, Uttarakhand, ${ }^{3}$ Reader, Dept. of Prosthodontics, \\ Seema Dental College \& Hospital, Rishikesh, Uttarakhand, India
}

*Corresponding Author: Yogeshwari Krishnan

Email: dryogeshwari1@gmail.com

\begin{abstract}
Various studies have been conducted so far using oral infection to be a hypothesis saying it to be a cause of Cardiovascular Diseases. Periodontal infections being the leading source but the conflict still continues to linger, that there is dental contribution towards heart problems. It is undetermined, whether this relationship is coincidental or specific. Though most studies have concluded it to be one positive relationship.
\end{abstract}

Keywords: Oral health, Heart health, Periodontal diseases and epidemiology.

\section{Introduction}

Cardiovascular disease is one of the leading causes of chronic disease morbidity and mortality in industrialised countries. According to the World Health Organisation, $31 \%$ of all deaths globally are due to CVD. Contributing risk factors of CVD include family history, diabetes, hypertension, hyperlipidaemia, tobacco use, limited physical activity, obesity and poor dietary intake. Additionally, there is also growing evidence that another potential risk factor for CVD is periodontal disease. Periodontal diseases are infectious and inflammatory and cause a risk towards heart attacks and strokes as shown through many studies. This article is regarding the association between dental infections and CVD mainly considering periodontal infections. ${ }^{1}$

\section{Literature Review}

In 1989, an old report relating to chronic infections with vascular diseases proposed by French \& German scientists was received by two Scandinavian reports. ${ }^{2}$ Mattila \& colleagues $^{3}$ found higher levels of periodontitis, caries, apical lesions, pericoronitis etc. in patients who had a recent history of myocardial infarction than those who were normal and healthy from the same population.

While Syrjanen and colleagues ${ }^{4}$ had found greater oral health issues among patients of recent stroke. A careful conclusion was drawn between the risk factors of CVD and Periodontal diseases, like old age, males, cigarette smoking, diabetes \& poor socio economic status. Confounding was done this way. ${ }^{5}$ Enrolment patients was done when they got hospitalized due to any heart conditions to note a negative effect of it on their oral health but the geographical homogeneity and less number of participants enrolled in these studies precluded any reliable generalizations beyond the specific study population. ${ }^{1}$

On the other hand a few studies were being conducted to overcome these limitations and there about 100,000 adults both males and females from varied population areas were taken which enhanced the consistency and generalizability of the proposed association between periodontal disease and CVD. ${ }^{1}$

But all studies don't support this hypothesis. The reports from the Health Professionals Follow-Up Study 6 and the Physicians' Health Study ${ }^{7}$ have shown no relation between periodontal health \& CVD \& Stroke. This was a large population survey consisting of 66,000 male health professionals but the drawback was that the participants were asked through self-filled questionnaires rather than via clinical examination.

Also Hujoel and colleagues ${ }^{8}$ found no existing association between periodontal disease and coronary heart disease in the First National Health and Nutrition Examination Survey (NHANES I) cohort. $\mathrm{Wu}$ and colleagues $^{9}$ though had found strong positive associations between periodontal disease and stroke in the same NHANES population in which Hujoel and colleagues ${ }^{10}$ did not.

Joshipura and colleagues reported no association between periodontal disease and coronary disease,${ }^{6}$ but between periodontal disease and Stroke.

Oral Infections and Vascular Disease Epidemiology Study (INVEST) $)^{11,12}$ and Study of Health in Pomerania(SHIP) ${ }^{13}$ suggested from their data that edentulous people remain at a higher risk or a potentially intermediate risk of developing subclinical CVD. It is also possible that the potential risk from periodontal infections might not be reversible after a certain threshold of subclinical CVD has developed.

Some reports from United States ${ }^{9} \&$ Germany $^{14}$ have said that there is association between periodontal diseases and CVD more in males ${ }^{12}$ In SHIP, Desvarieux and colleagues ${ }^{13}$ reported that men had more severe periodontal disease than women. Thus a moderate association is known to exist between CVD and Periodontal diseases as assessed radiographically and clinically. 
Oral Health Contributors towards Heart Diseases ${ }^{15}$ Periodontal Diseases: Periodontal Diseases cause inflammation. Inflammation being an immune response and can be beneficial when your body is fighting off infection, but chronically high levels effects health conditions causing cardiovascular diseases.

Invasive Dental Procedures: Surgery can increase inflammation. The researchers thus have said invasive dental procedures, particularly the treatment for periodontal diseases, increases the risk of heart attack and stroke because they boost the inflammation levels in the body as it responds to bacteria entering the bloodstream following surgery.

Surgical Removal of Teeth/Surgical Extractions: The study was based on records of 1,152 Medicare patients diagnosed with a heart attack or stroke between 2002 and 2006, and also had an invasive dental procedure during that time period. The most common procedure was the surgical extraction.

Sharing Risk Factors: Periodontitis and heart disease share risk factors such as smoking, age and diabetes, and both contribute to inflammation in the body. Although these shared risk factors may explain why diseases of the blood vessels and mouth can occur simultaneously.
Already Existing Heart Conditions: In cases of preexisting heart conditions, there can be a risk of developing bacterial endocarditis an infection of the heart's valves. When there is bleeding in the mouth, certain oral bacteria like P.Gingivalis and S.Sanguins enter the blood stream and settle onto these abnormal heart valves or tissue which are weakened by an existing heart condition. The infection can thus damage or even destroy heart valves or tissues.

There are some precautions which need to be taken in case of any of the following conditions ${ }^{16}$ : Artificial (prosthetic) heart valves, A history of endocarditis, Congenital heart, Heart valve defects or damaged Heart valves (scarred) by conditions such as rheumatic fever, Mitral valve prolapse with a murmur, Hypertrophic cardiomyopathy

\section{Pathophysiology of CVD Caused by Periodontitis ${ }^{17}$}

Regular, proper brushing and flossing help removes food remnants and thus bacteria from our teeth. If not brushed correctly, these bacteria can cause gum inflammation, i.e. gingivitis and, if left untreated, causes periodontitis or periodontal diseases.

The mechanism by which Periodontitis may be related to heart diseases is depicted below in the fig. ${ }^{18}$

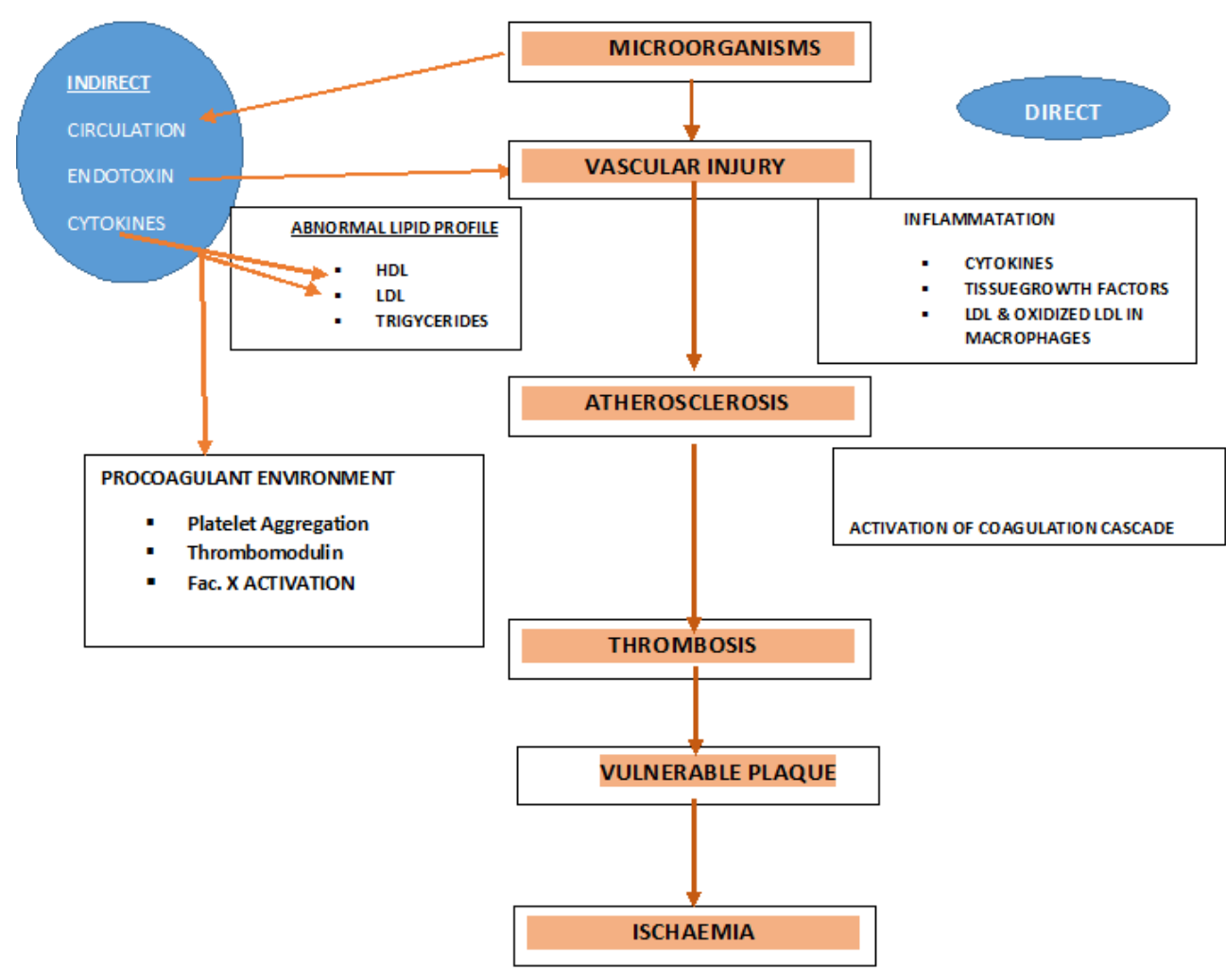

Fig. 1

When periodontitis occurs, the gums pull away from the teeth, creating space beneath the gum line where the bacteria flourish. Bacteria can easily enter the bloodstream and travel to the heart's arteries, harden them and cause atherosclerosis. The plaque grows on the arteries inner walls and restricts the blood flow 
throughout the body thus causing coronary artery disease. ${ }^{17}$

There are some theories suggesting how the bacteria from the oral cavity cause arteriosclerosis. Giacona and colleagues ${ }^{19}$ found that certain strains of P. Gingivalis are capable of infecting macrophages and enhancing foam cell formation in the vascular wall thus accelerating atherosclerotic process. In vitro studies have demonstrated the ability of Streptococcus Sanguis and $\mathrm{P}$. gingivalis to induce platelet aggregation and hypercoagulability increasing the formation of thrombus, which can lead to ischemic cardiovascular diseases. ${ }^{20-23}$ Some medical experts believe the oral bacteria triggers the body's inflammatory response to infection and causes blood cells to swell, which may narrow the heart's arteries. One other theory suggests that the oral bacteria adheres to the fatty plaques in the bloodstream, causing blockages. ${ }^{17}$

Data from the Third National Health and Nutrition Examination Survey found higher levels of C-reactive protein and fibrinogen, which are cardiovascular risks, are higher in people with gum diseases. Regular dental check-ups and treating them at spot decreases the $\mathrm{C}$ Reactive Protein levels which are seen mostly in people being treated for periodontal diseases..$^{24-28}$

Gums bleed when they are inflamed during biting, brushing etc. But this bleeding can lead to a potentially fatal heart condition called endocarditis. This is an infection caused due to the growth of bacteria in the inner lining of the heart. This infection prevents the heart's valves from functioning efficiently which may lead to heart failure. ${ }^{17}$

Other probable mechanism linking oral infections with CVD is the molecular mimicry, in which the antibodies targeted toward bacterial species unintentionally cross-react with host cells. Like in heat shock protein 60 has been studied for its possible role in mediating infection-induced atherosclerosis. ${ }^{29}$ These illustrations of false identity could so be leading to vascular inflammation and atherosclerosis.

\section{Treatment \& Prevention}

For treatment of periodontitis, the most important strategy is the effective control of the supragingival and subgingival plaques through active promotion of and meticulous attention to oral hygiene. Discourage frequent snaking or foods which are carbohydrate-rich or intake of sugar containing beverages. Chlorhexidine acts as cationic detergent that kills a wide range of bacteria and is retained on the oral surfaces for prolonged periods to prevent plaque advancements. Certain types of severe periodontitis are amenable to systemic antimicrobial therapy in conjunction with mechanical debridement (scaling and root planning). This protocol has often obviate the need for radical surgical resection of periodontal tissues. In various clinical studies of advance periodontitis, systemic metronidazole (500 mg PO TDS) or doxycycline (200 mg PO BD) for 1 to 2 weeks in conjunction with rigorous mechanical debridement of the root surfaces.

Prevention: The need for definitive restoration or extraction of the infected tooth, the primary source of odontogenic infection is readily apparent. Deep periodontal scaling and endodontic treatment with root filling are required. The key for the prevention of advanced periodontitis is the active promotion of oral hygiene that include: a) rigorous brushing and dental flossing after each meal (b) dietary counselling to reduce the indigestion of carbohydrates-rich foods or beverages (c) use of topical fluorides and oral antimicrobial rinses such as chlorhexidine for patients at high risk for dental caries (d) behavioural modification of risk factors, such as tobacco smoking (e) overcoming the reluctance for regular visits to dental professionals.Thus, if we want healthy heart quit smoking, manage weight, control blood pressure, stay active, get regular medical \& dental check-up done while maintaining a good oral hygiene and oral health. ${ }^{30}$

\section{Conclusion}

It's not the first time that there is discussion over the link between oral health and the general overall health of a person. There is strong evidence present which supports linkage between oral and general health. Many systemic diseases have oral manifestations as an initial sign and oral complications of many systemic diseases compromise quality of life. The control of oral infections is an important precaution to improve oral health, overall well-being and prevent health complications. So while you take care of your teeth you are also taking care of your entire body!

\section{References}

1. Ryan T. Demmer, PhD; Moïse Desvarieux, MD, PhD; Periodontal infections and cardiovascular disease; JADA. 2006;137:155-205.

2. Nieto FJ. Infections and atherosclerosis: new clues from an old hypothesis? Am J Epidemiol. 1998;148(10):93748.

3. Mattila KJ, Nieminen MS, Valtonen VV. Association between dental health and acute myocardial infarction. BMJ. 1989;298(6676): 779-81.

4. Syrjanen J, Peltola J, Valtonen V, Iivanainen M, Kaste M, Huttunen JK. Dental infections in association with cerebral infarction in young and middle-aged men. $J$ Intern Med. 1989;225(3):179-84.

5. Rothman KJ, Greenland S. Modern epidemiology. Philadelphia: Lippincott-Raven; 1998:62.

6. Joshipura KJ, Rimm EB, Douglass CW, Trichopoulos D, Ascherio A, Willett WC. Poor oral health and coronary heart disease. J Dent Res. 1996; 75(9):1631-6.

7. Howell TH, Ridker PM, Ajani UA, Hennekens CH, Christen WG. Periodontal disease and risk of subsequent cardiovascular disease in U.S. male physicians. J Am Coll Cardiol. 2001;37(2):445-50.

8. Hujoel PP, Drangsholt M, Spiekerman C, DeRouen TA. Periodontal disease and coronary heart disease risk. JAMA. 2000;284(11):1406-10. 
9. Wu T, Trevisan M, Genco RJ, Dorn JP, Falkner KL, Sempos CT. Periodontal disease and risk of cerebrovascular disease: the first national health and nutrition examination survey and its follow-up study. Arch Intern Med. 2000;160(18):2749-55.

10. Hujoel PP, Drangsholt M, Spiekerman C, Derouen TA. Examining the link between coronary heart disease and the elimination of chronic dental infections. JADA. 2001;132(7):883-9.

11. Desvarieux M, Demmer RT, Rundek T. Relationship between periodontal disease, tooth loss, and carotid artery plaque: the Oral Infections and Vascular Disease Epidemiology Study (INVEST). Stroke. 2003;34(9):2120-5

12. Engebretson SP, Lamster IB, Elkind MS. Radiographic measures of chronic periodontitis and carotid artery plaque. Stroke. 2005;36(3):561-6.

13. Desvarieux M, Schwahn C, Volzke H. Gender differences in the relationship between periodontal disease, tooth loss, and atherosclerosis. Stroke. 2004;35(9):2029-35.

14. Grau AJ, Becher H, Ziegler CM. Periodontal disease as a risk factor for ischemic stroke. Stroke. 2004;35(2):496501.

15. Increase Risk of Heart Attack; by Rachael Rettner, Senior Writer; October 18, 2010 05:01pm ET https://www.livescience.com/35065-dental-proceduresincrease-risk-heart-attack-stroke-101018.html

16. How Oral Health And Heart Disease Are Connected, by Tracey Sandilands https://www.colgate.com/en-us/oralhealth/conditions/heart-disease/heart-disease-and-gumdisease

17. Can bad teeth lead to a bad heart? The Guardian Life Insurance Company of America (Guardian), New York, NY. File \#2017-47183 (exp. 10/19)

18. Fong IW. Infections and their role in atherosclerotic vascular disease. JADA. 2002;133(supplement):7S-13S.

19. Giacona MB, Papapanou PN, Lamster IB. Porphyromonas gingivalis induces its uptake by human macrophages and promotes foam cell formation in vitro. FEMS Microbiol Lett. 2004;241(1):95-101.

20. Herzberg MC, Meyer MW. Effects of oral flora on platelets: possible consequences in cardiovascular disease. J Periodontol. 1996;67(supplement 10):1138-42.
21. Meyer MW, Gong K, Herzberg MC. Streptococcus sanguis-induced platelet clotting in rabbits and hemodynamic and cardiopulmonary consequences. Infect Immun. 1998;66(12):5906-14.

22. Fong IW. Emerging relations between infectious diseases and coronary artery disease and atherosclerosis. CMAJ. 2000;163(1):49-56.

23. Imamura T, Potempa J, Tanase S, Travis J. Activation of blood coagulation factor $\mathrm{X}$ by arginine-specific cysteine proteinases (gingipain-Rs) from Porphyromonas gingivalis. J Biol Chem. 1997;272(25):16062-7.

24. Slade GD, Ghezzi EM, Heiss G, Beck JD, Riche E, Offenbacher S. Relationship between periodontal disease and C-reactive protein among adults in the Atherosclerosis Risk in Communities study. Arch Intern Med. 2003;163(10):1172-9.

25. Slade GD, Offenbacher S, Beck JD, Heiss G, Pankow JS. Acutephase inflammatory response to periodontal disease in the US population. J Dent Res. 2000;79(1):49-57.

26. Loos BG, Craandijk J, Hoek FJ, Wertheim-van Dillen PM, van der Velden U. Elevation of systemic markers related to cardiovascular diseases in the peripheral blood of periodontitis patients. $J$ Periodontol. 2000;71(10):1528-34

27. Ebersole JL, Machen RL, Steffen MJ, Willmann DE. Systemic acute-phase reactants, C-reactive protein and haptoglobin, in adult periodontitis. Clin Exp Immunol. 1997;107(2):347-52.

28. Kowolik MJ, Dowsett SA, Rodriguez J, De La Rosa RM, Eckert GJ. Systemic neutrophil response resulting from dental plaque accumulation. J Periodontol. 2001;72(2):146-51.

29. Ford P, Gemmell E, Walker P, West M, Cullinan M, Seymour G. Characterization of heat shock proteinspecific T cells in atherosclerosis. Clin Diagn Lab Immunol. 2005;12(2):259-67.

30. Dental Health and Heart Healthhttp://www.heart.org/HEARTORG/HealthyLiving/ Dental-Health-and-HeartHealth_UCM_459358_Article.jsp\#.Wvsu44iFPIU 